\title{
Overall similarity, natural properties, and paraphrases
}

\author{
Ghislain Guigon
}

Published online: 6 February 2013

(C) Springer Science+Business Media Dordrecht 2013

\begin{abstract}
I call anti-resemblism the thesis that independently of any contextual specification there is no determinate fact of the matter about the comparative overall similarity of things. Anti-resemblism plays crucial roles in the philosophy of David Lewis. For instance, Lewis has argued that his counterpart theory is anti-essentialist on the grounds that counterpart relations are relations of comparative overall similarity and that anti-resemblism is true. After Lewis committed himself to a form of realism about natural properties he maintained that anti-resemblism is true about the relations of overall similarity that enter his counterpart theory and his analysis of counterfactuals. However, in this article I argue that Lewis's account of degrees of naturalness for properties combined with his modal realism entails that anti-resemblism is false. The Lewisian must amend Lewis's system if she aims to benefit from the alleged virtues of anti-resemblism. I consider two ways of amending it, neither of which is a free lunch.
\end{abstract}

Keywords David Lewis · Overall similarity · Natural properties · Modal realism

I might be likely to say that hippos are more similar to pigs than to whales. Hippos and pigs are physically similar in several respects and belong to the group of eventoed ungulates, which excludes cetaceans. But whales are the closest living relatives of hippos-they diverged from cetaceans about fifty-five million years ago-and I am interested in the evolution of species. Circumstances appear to alter our judgments of comparative overall similarity (hereafter, overall similarity). As a result, there is a prima facie case for the following thesis:

G. Guigon ( $₫)$

Département de Philosophie, Université de Genève, 2, rue de Candolle, 1211 Genève 4, Switzerland e-mail: ghislain.guigon@unige.ch 
Anti-resemblism. Independently of any contextual specification, there is no determinate fact of the matter about the overall similarity of things (about the truth value of the propositional content of judgments of overall similarity).

The opposite thesis is the following:

Resemblism. Independently of any contextual specification, there is a determinate fact of the matter about the overall similarity of things (about the truth value of the propositional content of judgments of overall similarity).

The debate between resemblism and anti-resemblism is analogous to the debate between essentialism - the view that there is a determinate fact of the matter about an object's modal properties (about the truth value of de re modal propositions) not relative to specifications-and anti-essentialism. ${ }^{1}$ But the two debates are more closely related. For David Lewis has argued that his counterpart theory is antiessentialist on the grounds that anti-resemblism is true:

I am by no means offering a wholehearted defence of "Aristotelian essentialism." For the essences of things are settled only to the extent that the counterpart relation is, and the counterpart relation is not very settled at all. Like any relation of comparative overall similarity, it is subject to a great deal of indeterminacy (1) as to which respects of similarity and difference are to count at all, (2) as to the relative weights of the respects that do count, (3) as to the minimum standard of similarity that is required, and (4) as to the extent to which we eliminate candidates that are similar enough when they are beaten by competitors with stronger claims. Further, (...) the vagueness of the counterpart relation-and hence of essence and de re modality generally-may be subject to pragmatic pressures, and differently resolved in different contexts. ${ }^{2}$

Anti-resemblism plays crucial roles in Lewis's philosophy. Relations of overall similarity enter his system in two guises. First, there is the relation of overall similarity between possibilia - $x$ is similar to $y$ and $x$ is at least as similar to $y$ as any other part of $x$ 's possible world - the so-called counterpart relation, that is a primitive of his counterpart theory. ${ }^{3}$ According to Lewis, the indeterminacy of the counterpart relation adequately accounts for the indeterminacy of the truth value of de re modal propositions. ${ }^{4}$ But also it allows us to solve problems of material constitution without being committed to numerically distinct coincident things or to the contingent identity of a thing with itself. ${ }^{5}$ Further, there is a relation of overall similarity among worlds - $w$ is at least as similar to $w_{1}$ as is $w_{2}$ - that governs counterfactuals. ${ }^{6}$ Lewis argues that the indeterminacy of this relation adequately matches that of counterfactuals themselves. ${ }^{7}$

\footnotetext{
1 See e.g. Quine (1961, p. 155), Paul (2004).

2 Lewis (1983b, p. 42).

3 See Lewis (1983a, c).

4 See Lewis (1983b, pp. 42-43).

5 See Lewis (1983c, pp. 51-52).

6 See Lewis (1973, p. 48).

7 Lewis (1973, pp. 93-94).
} 
After Lewis committed himself to realism about natural properties in his "New Work for a Theory of Universals" he maintained that anti-resemblism is true about the relations of overall similarity that enter his counterpart theory and his analysis of counterfactuals. ${ }^{8}$ However, in this article I shall argue that Lewis's doctrine of natural properties combined with his modal realism yields a specific version of resemblism. The Lewisian must amend Lewis's system if she aims to benefit from the alleged virtues of anti-resemblism. I conclude by considering two ways of amending it, neither of which is a free lunch.

\section{Argument for anti-resemblism}

Whenever things are similar in some respect they either share a common property or have similar properties. Let us say that a property is a respect of similarity if and only if sharing this property or having a property similar to it counts when evaluating whether things are similar to each other. Likewise, a respect of dissimilarity is any property such that lacking it or having a property dissimilar to it counts when evaluating whether things are dissimilar. Lewis conceives of a relation of overall similarity as any weighted resultant of respects of similarity and dissimilarity. ${ }^{9}$

Anti-resemblists like Goodman and the young Lewis typically maintain that each of the following claims is true:

\section{Abundance Properties are abundant.}

Neutrality Independently of any contextual specification (for short, objectively), properties are all on a par with regards to their ability to make for similarity and dissimilarity, i.e. no property is more or less of a respect of similarity and dissimilarity than any other property is.

Discrimination Overall similarity is discriminating, i.e. things are not equally similar or dissimilar to each other. ${ }^{10}$

According to Lewis, any class of actual and otherworldly things is a property. Hence properties are extremely abundant in his ontology. ${ }^{11}$ On the other hand, Neutrality follows from the plausible assumption that properties, conceived of as classes of things, have the same ontological status and are thus on a par with regards to their ability to count in similarity-making. Finally, Discrimination is based on the common-sense conviction "that we undeniably do make judgments of comparative similarity". ${ }^{12}$ If Discrimination were false, there would be no point making such judgments.

\footnotetext{
${ }^{8}$ See Lewis (1986a, p. 252; 1986b, p. 52).

9 Lewis (1986b, pp. 53-54).

${ }^{10}$ Goodman (1972, pp. 443-445) and Lewis (1973, pp. 91-92).

11 Lewis (1999a, p. 10).

12 Lewis (1986b, p. 53).
} 
Given Abundance any two things have in common and fail to share the same infinite amount of properties as any further two things. ${ }^{13}$ If so, a necessary condition for Discrimination to be true is that there be a selection between these properties that count for similarity and dissimilarity and those that do not count. Given Neutrality we cannot expect this selection to be an objective matter. Hence the required selection must be grounded in the way things are represented when comparing them in judgments of overall similarity. Since the way things are represented in judgments of overall similarity is indeterminate and context-dependent, overall similarity is an indeterminate and context-sensitive matter. Therefore, if Abundance, Neutrality, and Discrimination are true, so is anti-resemblism. ${ }^{14}$

\section{Realism about natural properties}

Assuming that Discrimination is true resemblists can deny either Abundance or Neutrality in order to block the preceding argument in favour of anti-resemblism. Some resemblists follow David Armstrong who denies Abundance and maintains that properties are sparse. ${ }^{15}$ But resemblists can agree with Abundance and instead appeal to Lewis's doctrine of natural properties in order to reject Neutrality. For, according to Lewis's doctrine, while most properties are "undiscriminating" and have "nothing to do with similarity", there is an élite minority of properties, namely the natural ones, that "capture facts of resemblance" and "make for qualitative similarity". "Since, according to Lewis, the difference between natural and merely abundant properties is an objective one, ${ }^{17}$ it follows that properties are objectively not all on a par with regards to their ability to make for similarity. Hence Neutrality is false.

By committing himself to an objective difference between natural and merely abundant properties Lewis thereby rejected Neutrality. Nevertheless, he maintained that anti-resemblism is true. Indeed although the idea of natural properties as being these properties that carve reality at the joints is closely linked to the belief in objective similarity, ${ }^{18}$ realism about natural properties, and a fortiori the rejection of Neutrality, is compatible with anti-resemblism. For if natural properties are still quite abundant and are all on a par with regards to their ability to make for qualitative similarity, Discrimination can fail. Yet natural respects of similarity are very abundant in Lewis's system. Take any arbitrary thing, $a$, and form the disjunctive property $F$ or $G$, where $F$ is one of $a$ 's most natural properties and $G$ is

\footnotetext{
13 See Goodman (1972, pp. 443-444) and Lewis (1986b, p. 53).

14 Notice that this traditional argument in favour of anti-resemblism does not take into account the role played by the similarity of properties in the evaluation of overall similarity. However, the similarity of properties plays a central role in my argument to the conclusion that Lewis's mature doctrine yields a form of resemblism.

15 See e.g. Armstrong (1978, pp. 7-94).

16 See Lewis (1999a, p. 13; 1986a, pp. 59-60). Notice that in this article I follow Lewis in using "resemblance" and "similarity" synonymously.

17 See Lewis (1999a, p. 14).

18 See e.g. Sider (2011, pp. 1-8).
} 
an arbitrary perfectly natural property. ${ }^{19}$ Thus described, $F$ or $G$ is a fairly natural property had by $a$. Then consider the infinity of possible worlds and the infinite variations in perfectly natural properties, resident or alien, exemplified in distinct worlds. $^{20}$ This should give a rough idea of the large infinity of natural properties alike to $F$ or $G$ that $a$ shares with every $F$-thing. So the rejection of Neutrality does not suffice to yield resemblism. However, I shall argue that Lewis's doctrine of natural properties combined with his modal realism entails resemblism.

According to Lewis, natural properties are not all on a par. Some are more natural than others, and some are perfectly natural-they are such that no property is more natural than them. ${ }^{21}$ Perfectly natural properties and relations carve reality at its most natural joints and nowhere else, they are perfectly determinate and specific, the sets of their bearers are ipso facto not at all miscellaneous, and there are just enough of them to characterise things completely and without redundancy. ${ }^{22}$ Properties that are not perfectly natural are definable in terms of Boolean operations on perfectly natural ones. Boolean constructs are not all on a par: some are natural, some are not natural, and, among the natural ones, some are more natural than others. Naturalness comes by degrees, and degrees of naturalness are an objective matter.

But how does Lewis account for degrees of naturalness? Commentators commonly account for degrees of naturalness as a mere function of the relative complexity of the way less-than-perfectly natural properties are defined out of their perfectly natural basis. ${ }^{23}$ But Nolan rightly acknowledges that this account is unsatisfactory for the following reason. ${ }^{24}$ Some natural properties of the same degree of complexity can be more or less gerrymandered. For instance, the property definable as carmine or vermillion is less gerrymandered than the property definable as carmine or azure. But if degrees of naturalness are a mere function of the relative complexity of the way properties are defined out of their perfectly natural basis, these properties are equally natural, which seems wrong.

However, these commentators misrepresent Lewis's account. When Lewis describes how universals should help us in recognising the natural properties, he explicitly claims that less-than-perfectly natural properties are "made so by families of suitable related universals". ${ }^{25}$ The nature of the suitable relation is made clear in the attached footnote:

Here I assume that some solution to the problem of resemblance of universals is possible, perhaps along the lines suggested by Armstrong in Universals, II, pp. 48-52 and 101-131; and that such a solution could be carried over into a

\footnotetext{
19 See below on degrees of naturalness.

${ }^{20}$ Lewis (1986a, pp. 159-165).

21 Lewis (1999a, pp. 13-14).

22 Lewis (1986a, pp. 59-60).

23 See, for instance, Hall (2010), Nolan (2005, p. 24), and Taylor (2006, p. 104).

24 Nolan (2005, p. 24).

25 Lewis (1999a, p. 13).
} 
theory of resemblance of perfectly natural properties, even if we take naturalness of properties as primitive. ${ }^{26}$

The problem of resemblance of universals is the problem of accounting for the fact that universals resemble one another to various degrees. ${ }^{27}$ So here Lewis explicitly draws a link between less-than-perfectly natural properties and similarities to various degrees between perfectly natural properties. Accordingly, the right account of degrees of naturalness is two-dimensional: one dimension is a function of the relative complexity of the way less-than-perfectly natural properties are defined out of their perfectly natural basis; but there is a second dimension, which is a function of the relative similarity of these perfectly natural properties (or of their corresponding universals) in terms of which less-than-perfectly natural properties are defined. Contrary to what commentators usually do, we should not focus on the first dimension if we aim to understand Lewis's account properly.

Lewis maintains that degrees of naturalness are an objective, context-insensitive, and determinate matter. But Lewis partly grounds the hierarchy of degrees of naturalness of less-than-perfectly natural properties in the relative similarity of these perfectly natural properties in terms of which less-than-perfectly natural properties are defined. Therefore, Lewis is committed to the claim that similarity of perfectly natural properties, and $a$ fortiori of less-than-perfectly natural properties that are defined in terms of perfectly natural ones, is a context-insensitive and determinate matter. ${ }^{28}$ This means that, in Lewis's doctrine of natural properties, there are propositions of the following forms that are determinately true independently of any contextual specification:

(1) $F$ is more similar to $G$ than it is to $H$;

(2) $F$ is as similar to $G$ as it is to $H$;

where ' $F$ ', ' $G$ ', and ' $H$ ' stand for natural properties. ${ }^{29}(1)$ is true for any values of ' $F^{\varsigma}$, ' $G$ ', and ' $H$ ' such that $F$ is similar to both $G$ and $H$ but is more similar to $G$ than to $H$. Intuitively, carmine is thus related to Vermillion and azure. But (1) is also true for any values of ' $F$ ', ' $G$ ', and ' $H$ ' such that $F$ is similar to $G$ but not similar to $H$. Intuitively, carmine is thus related to vermillion and triangularity. On the other

\footnotetext{
${ }^{26}$ Lewis (1999a, pp. 13-14, note 7). Notice that by rejecting Armstrong's structural universals in (Lewis 1999b) Lewis thereby rejected Armstrong's solution to the problem of resemblance of universals, which consists in conceiving of resembling universals as structural ones. However, this does not mean that Lewis changed his mind on the link between degrees of naturalness and the similarity of perfectly natural properties. He might have preferred another account of this link. For instance, an account that consists in taking modal realist paraphrases for predications of similarity to properties (see below) as genuine analyses.

27 "The different shapes resemble each other: they are all shapes. Furthermore, one (sort of) shape may resemble another more closely than it resembles a third. Triangularity is more like quadrilaterality than it is like circularity. (...) Our task is to give an account of resemblances such as these." Armstrong (1978, p. 101); see also Eddon (2007).

28 If the similarity of natural properties is not determinate or fine-grained, then the relative naturalness of some natural properties is not fine-grained either, given the link Lewis draws between similarity of natural properties and degrees of naturalness. But the Lewisian denies that the joints of nature are indeterminate. So the Lewisian must agree that the similarity of natural properties is a determinate matter.
}

29 (1) and (2) could be taken as primitives or as derived from " $F$ is at least as similar $G$ as it is to $H$ ". 
hand, (2) is true for any values of ' $F$ ', ' $G$ ', and ' $H$ ' such that $G$ and $H$ are both similar to $F$ and are equally similar to $F$. One may think, for instance, that green is thus related to yellow and blue. But (2) is also true whenever ' $F$ ', ' $G$ ', and ' $H$ ' are such that neither of $G$ and $H$ is similar to ' $F$ '. This seems to be the case, for instance, whenever $F$ stands for a particular shade of colour, while ' $G$ ' and ' $H$ ' stand for determinate masses, electric charges, shapes, etc.

But how is relative similarity of natural properties linked to relative naturalness of less-than-perfectly natural properties? Lewis does not tell us. So we can only speculate as to how relative similarity of natural properties should be linked to relative naturalness in order to yield an adequate theory of natural properties. Following Nolan, a theory of natural properties that fails to acknowledge that carmine or vermillion is more natural than carmine or azure is inadequate. So I propose that comparative similarity of natural properties should be linked to relative naturalness of less-than-perfectly natural properties so as to make the following true:

(*) If $F$ is more similar to $G$ than it is to $H$, the property definable as $F$ or $G$-alternatively, $F$ and $G$-is more natural than the property definable as $F$ or $H$-alternatively, $F$ and $H$;

where ' $F$ ', ' $G$ ', and ' $H$ ' stand either for perfectly natural properties or for properties whose definitions in terms of perfectly natural properties are equally complex. Given (*), carmine or vermillion turns out more natural than carmine or azure.

But (*) should be supplemented with the following principle in order to close further gaps in the ordering of less-than-perfectly natural properties:

(**) If $F$ is as similar to $G$ as it is to $H$, the property definable as $F$ or $G$-alternatively, $F$ and $G$-is as natural as the property definable as $F$ or $H$-alternatively, $F$ and $H$

where ' $F$ ', ' $G$ ', and ' $H$ ' stand either for perfectly natural properties or for properties whose definitions in terms of perfectly natural properties are equally complex. Consider six natural properties whose definitions in terms of perfectly natural properties are equally complex- $F^{1}, F^{2}, F^{3}, F^{4}, G^{1}$, and $G^{2}$-and assume the following about them. $F^{2}$ and $F^{3}$ are both similar to $F^{l}$ but are such that $F^{2}$ is more similar to $F^{1}$ than $F^{3}$ is. $F^{3}$ and $F^{4}$ are both similar to $F^{1}$, and $F^{3}$ is as similar to $F^{1}$ as $F^{4}$ is. Finally, $F^{l}, G^{l}$, and $G^{2}$ are such that neither of $G^{1}$ and $G^{2}$ is similar to $F^{l}$. This situation can be represented as follows (where points represent natural properties, where the region within the circle represents the domain of properties that are similar to $F^{l}$, and where distance to $F^{l}$ represents similarity distance to $F^{l}$ ) (Fig. 1).

Since these properties are equally complex natural properties the view that relative naturalness is a mere function of the relative complexity of properties yields the following ordering: $F^{1}$ or $F^{2}, F^{1}$ or $F^{3}, F^{1}$ or $F^{4}, F^{1}$ or $G^{1}$, and $F^{1}$ or $G^{2}$ are equally natural properties. This seems wrong. $F^{l}$ or $F^{2}$ carves reality at the joints better than each of $F^{1}$ or $F^{3}, F^{1}$ or $F^{4}, F^{1}$ or $G^{1}$, and $F^{1}$ or $G^{2}$ does; $F^{1}$ or $F^{3}$ and $F^{1}$ or $F^{4}$ carve reality at the joints equally well and carve it better at the joints than both 
Fig. 1 Comparative similarity to $F^{1}$

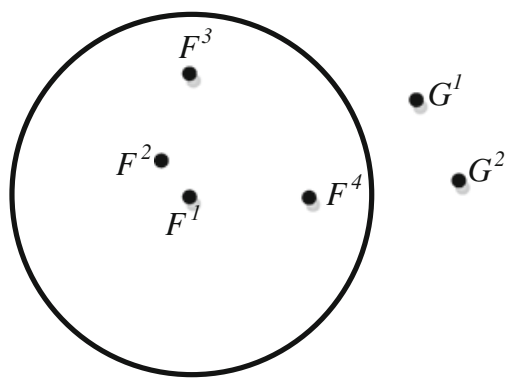

$F^{l}$ or $G^{1}$ and $F^{l}$ or $G^{2}$; finally, $F^{l}$ or $G^{l}$ and $F^{l}$ or $G^{2}$ carve reality equally badly at the joints. Yet if the Lewisian assumes with Lewis that there is a link between objective facts of comparative similarity for natural properties and degrees of naturalness, she can maintain $(*)$ and $(* *)$ in order to derive the desired ordering: $F^{l}$ or $F^{2}$ is more natural than each of $F^{l}$ or $F^{3}, F^{l}$ or $F^{4}, F^{l}$ or $G^{l}$, and $F^{l}$ or $G^{2} ; F^{l}$ or $F^{3}$ and $F^{1}$ or $F^{4}$ are equally natural and are more natural than $F^{1}$ or $G^{1}$ and $F^{1}$ or $G^{2}$; $F^{l}$ or $G^{l}$ and $F^{l}$ or $G^{2}$ are equally natural.

\section{Modal realist paraphrases}

Lewis believes that his ontology of possible worlds obeys a principle of completeness according to which, roughly, there is no gap in the logical space and so every possibility is realised. ${ }^{30}$ And he claims that the completeness of the modal pluriverse allows him to paraphrase away statements about the comparative similarity of properties. Thus

(3) Red is more similar to orange than it is to blue

can be paraphrased as

$\left(3^{\prime}\right)$ Some red thing is more similar to some orange thing than any red thing is similar to any blue thing

in virtue of the close similarity between red and orange, where the things in question need not be part of a same world. ${ }^{31}$ It is important to acknowledge that $\left(3^{\prime}\right)$ is a statement of overall similarity rather than a statement of similarity in some respect. For a cross-world comparison is not required in order to account for (3) in terms of comparative similarity of things with respect to colour. That (3) can be paraphrased as $\left(3^{\prime}\right)$ shows that such a paraphrase is available for any predication of comparative similarity to properties. So, for any $F, G$, and $H$

\footnotetext{
${ }^{30}$ Lewis (1986a, p 86). Although it is doubtful that completeness can be achieved, I shall assume it for the sake of my demonstration; see Divers and Melia (2002).

${ }^{31}$ Lewis (1999a, pp. 16-17, 1986a, p. 13).
} 
(1) $F$ is more similar to $G$ than it is to $H$

can be paraphrased as

$\left(1^{\prime}\right)$ Some $F$-thing is more similar to some $G$-thing than any $F$-thing is similar to any $H$-thing

where these things need not be part of a same world. Likewise, I propose that the modal realist can paraphrase

(2) $F$ is as similar to $G$ as it is to $H$

as

$\left(2^{\prime}\right)$ For any $F$-thing there is a $H$-thing such that the former is as similar to any $G$ thing as the latter is, and for any $H$-thing there is a $F$-thing such that the former is as similar to any $G$-thing as the latter is.

where these things need not be part of a same world.

Now, according to Lewis's doctrine of natural properties, some statements of the forms of (1) and (2) are objective truths since objective facts of comparative similarity for natural properties enter his account of degrees of naturalness. Suppose that (3) is such a truth that is not relative to any context. Then $\left(3^{\prime}\right)$ is an adequate paraphrase for (3) only if (3) and $\left(3^{\prime}\right)$ are true in the same conditions. So $\left(3^{\prime}\right)$, if it is an adequate paraphrase for (3), must be true not relative to any context. But $\left(3^{\prime}\right)$ expresses a determinate cross-world fact of overall similarity between things. So, according to the link Lewis draws between objective facts of similarity between properties and degrees of naturalness and given Lewis's claim that statements of the form of (1) can be adequately paraphrased as statements of the form of $\left(1^{\prime}\right)$, there are determinate facts of overall similarity between things that obtain independently of any contextual specification. Likewise, some statements of the form of (2) are objective truths. So if $\left(2^{\prime}\right)$ is a correct paraphrase for (2), some statements of overall similarity of the form of $\left(2^{\prime}\right)$ are objective truths in Lewis's system. But if so, resemblism is true.

In order to illustrate this result, suppose that colour charges are incompatible perfectly natural properties and that green charge is objectively more similar to blue charge than it is to red charge. Assuming with Lewis that $\left(1^{\prime}\right)$ is a correct paraphrase pattern for statements of the form of (1), we can derive that independently of any context some possible green quark, $a$, is more similar to some possible blue quark, $b$, than any possible green quark is similar to any possible red quark. So let us assume that $c$ is a possible red quark that is a near duplicate of $a$ and $b: a, b$, and $c$ are exactly similar in every perfectly natural respect except for their colour charges. ${ }^{32}$ Given the close similarity between $a$ and $c$, if one compares

\footnotetext{
32 Perfect duplicates share all their perfectly natural properties; near duplicates share almost all their perfectly natural properties; see Lewis (1999a, pp. 25-29).
} 
$a, b$, and $c$ in a context where no importance at all is assigned to colour charges one should judge that $b$ and $c$ are equally similar to $a$ (there is no reason to believe that there can be no such context). If so, according to Lewis's own claim about the relativity of overall similarity to contexts, it is true in the latter context that $b$ and $c$ are equally similar to $a$. However, my analysis reveals that, according to Lewis's realism about natural properties, this judgement must be false no matter what the context is. For the assumed close similarity between green and blue charges entails that independently of any contextual specification there is a determinate fact of the matter about the overall similarity of $a, b$, and $c: a$ is more similar to $b$ than it is to $c$.

Similarly, it can be shown that since there are objective truths of the form of (2) in Lewis's system and given the assumption that $\left(2^{\prime}\right)$ is a correct paraphrase pattern for statements of the form of (2), there are objective facts of equal similarity between possibilia. For instance, let us assume that $F^{1}, F^{2}$, and $F^{3}$ are incompatible perfectly natural properties such that, as an objective matter of fact, $F^{l}$ and $F^{2}$ are equally similar to $F^{3}$. Then let us assume that $i, j$, and $k$ are exactly similar in every perfectly natural respect except for the fact that $i$ has $F^{l}, j$ has $F^{2}$, and $k$ has $F^{3}$. Then it is true independently of any contextual specification that $i$ is as similar to $k$ as $j$ is.

\section{Applications}

If my reading of Lewis is correct, combining his doctrine of natural properties with his modal realism yields a version of resemblism: independently of any contextual specification, there is a similarity ordering of all things that is determined by objective similarity orderings between perfectly natural properties. This result is not without effect on Lewis's applications of relations of overall similarity that rely on their alleged indeterminacy.

Consider the quarks $a, b$, and $c$ again but this time assume that $a$ inhabits the actual world, while the only inhabitants of $w_{1}$ are a perfect duplicate of $b, b^{\prime}$, and a perfect duplicate of $c, c^{\prime}$. Since things could be the way they are in $w_{1}$ it follows by the modal realist principle of completeness that there is such a world as $w_{1}$. I have established that, on the assumption that green charge is objectively more similar to blue charge than it is to red charge, the link between degrees of naturalness and the similarity of perfectly natural properties combined with the assumption that $\left(1^{\prime}\right)$ is a correct paraphrase pattern for statements of the form of (1) entails that objectively $a$ is more similar to $b$ than it is to $c$. Since $b^{\prime}$ and $c^{\prime}$ are perfect copies of respectively $b$ and $c, b$ and $b^{\prime}$ are equally similar to $a$, and so are $c$ and $c^{\prime}$. Therefore, independently of any context $a$ is more similar to $b^{\prime}$ than it is similar to $c^{\prime}$. If so given Lewis's account of the counterpart relation, it is an objective fact that $b^{\prime}$ is a counterpart of $a$ in $w_{1}$, whereas $c^{\prime}$ is not a counterpart of $a$ in $w_{1}$. Since $b^{\prime}$ is a counterpart of $a$, Lewis's counterpart theory delivers the verdict that $a$ is possibly blue-charged. But since it is not the context, but objective facts of similarity between perfectly natural properties, that determine that $b^{\prime}$ is a counterpart of $a$, " $a$ is possibly blue-charged" is true independently of any contextual specification. So if my analysis of Lewis is correct, it shows that the addition of natural properties 
entails that facts about an object's de re modal properties are not indeterminate, contrary to Lewis's own claim. ${ }^{33}$

Assuming that natural properties of worlds stand in objective relations of similarity as well, a similar line of reasoning can be used to show that, given the link Lewis draws between degrees of naturalness and the similarity of natural properties and given the availability of modal realist paraphrases for statements of the form of (1) and (2), the relation of overall similarity that governs counterfactuals is not as context-sensitive as Lewis claims it is. ${ }^{34}$

\section{Conclusion}

The difficulty I explored in this article has emerged from the combination of the link Lewis draws between similarity of perfectly natural properties and degrees of naturalness with the assumption that $\left(1^{\prime}\right)$ and $\left(2^{\prime}\right)$ provide adequate paraphrase patterns for statements of the form of (1) and (2) respectively. So the Lewisian who aims to maintain anti-resemblism cum realism about natural properties can reject either the link between similarity of perfectly natural properties and degrees of naturalness or the availability of modal realist paraphrases for statements of the forms of (1) and (2).

Suppose she rejects the link between similarity of perfectly natural properties and degrees of naturalness and instead follows mistaken commentators in taking degrees of naturalness to be a mere function of the relative complexity of the way less-thanperfectly natural properties are defined out of their perfectly natural basis. Then, as I have argued following Nolan, her account of degrees of naturalness is intuitively inadequate. For intuitively the gerrymandered carmine or azure is not as natural as the non-gerrymandered carmine or vermillion. If the Lewisian maintains the link Lewis draws between similarity of perfectly natural properties and degrees of

\footnotetext{
33 Todd Buras (2006) has defended a similar conclusion. However, Buras's argument is based on the belief that, according to Lewis, shared perfectly natural properties (or shared maximally natural properties) are the privileged respects of similarity; see Buras (2006, pp. 35-36). From this belief, Buras concludes that the ontology assigns a privileged role to some specific relation of comparative overall similarity which is wholly determined by the number of shared perfectly natural properties independently of any contextual specification; see Buras (2006, pp. 32, 36, and 40,41). But my reading of Lewis and the model I gave show that the belief that shared perfectly natural properties are the privileged respects of similarity is wrong in Lewis's realism about natural properties. In Lewis's system, similarity of perfectly natural properties does not count less than commonality of perfectly natural properties in determining objective facts of overall similarity-otherwise $b^{\prime}$ and $c^{\prime}$ would be equally similar to $a$, which is not the case.

Notice that Buras would agree with me that, in the model I offered at the end of Sect. 3, it is true independently of any context that $i$ and $j$ are equally similar to $k$. However, the reason why Buras would maintain that $i$ and $j$ are equally similar to $k$ is different from mine and does not fit with Lewis's doctrine. Buras would conclude that $i$ and $j$ are equally similar to $k$ because $i$ and $j$ share the same number of perfectly natural properties with $k$. By contrast, according to my reading of Lewis, $i$ and $j$ are equally similar to $k$ because $i, j$, and $k$ are exactly similar in every perfectly natural respect except for the fact that $i$ has $F^{1}, j$ has $F^{2}$, and $k$ has $F^{3}$ and because $F^{1}$ and $F^{2}$ are equally similar to $F^{3}$.

34 The simplest model is the following. Consider three worlds, $w_{1}, w_{2}$, and $w_{3}$ that differ in exactly one natural respect: $w_{1}$ has $F^{1}, w_{2}$ has $F^{2}$, and $w_{3}$ has $F^{3}$. Then suppose that, objectively, $F^{1}$ is more similar to $F^{2}$ than it is similar to $F^{3}$.
} 
naturalness, she can account for the fact that carmine or vermillion is more natural than carmine or azure by embracing (*). But if she aims to drop this link, she needs to find an adequate substitute for (*) and (**). Such a substitute is still to be imagined.

On the other hand, if the Lewisian denies that statements of the form of (1) and (2) can be adequately paraphrased in terms of statements of the form of $\left(1^{\prime}\right)$ and $\left(2^{\prime}\right)$, then an alleged advantage of Lewis's analysis of modality over standard modal logic is lost. For, according to Lewis, while $\left(3^{\prime}\right)$ offers a correct paraphrase for the statement that a red thing could be more similar to an orange thing than any red thing could be similar to any blue thing, this modalised comparative cannot be expressed in standard modal logic. ${ }^{35}$ Moreover, the rejection of modal realist paraphrases for statements of the form of (1) and (2) deprives nominalists of the most satisfactory account of similarity statements between properties. ${ }^{36}$ I conclude that the Lewisian cannot have the alleged virtues of anti-resemblism for free.

Acknowledgments Thanks to Alexander Bown, Fabrice Correia, Filipe Drapeau Contim, Stephan Leuenberger, Kevin Mulligan, Frédéric Nef, the members of eidos (the Genevan research group in metaphysics), the audience and organisers of the conference "Another world is possible" on David Lewis in Urbino, and the editors and reviewer at Philosophical Studies for helpful comments on this paper or the ideas it presents.

\section{References}

Armstrong, D. M. (1978). Universals and scientific realism: A Theory of universals (Vol II). Cambridge: Cambridge University Press.

Buras, T. (2006). Counterpart theory, natural properties, and essentialism. Journal of Philosophy, 103(1), 27-42.

Divers, J., \& Melia, J. (2002). The analytic limit of genuine modal realism. Mind, 111, 15-35.

Eddon, M. (2007). Armstrong on quantities and resemblance. Philosophical Studies, 136(3), 385-404.

Goodman, N. (1972). Seven strictures on similarity. In N. Goodman (Ed.), Problems and projects (pp. 437-447). Indianapolis: Bobbs and Merrill.

Hall, N. (2010). Supplement on the natural/non-natural distinction to his 'David Lewis's metaphysics'. In EN Zalta (Ed.), The stanford encyclopedia of philosophy (Fall 2010 edition). http://plato. stanford.edu/archives/fall2010/entries/lewis-metaphysics.

Lewis, D. K. (1973). Counterfactuals. Oxford: Blackwell.

Lewis, D. K. (1983a). Counterpart theory and quantified modal logic. In D. K. Lewis (Ed.), Philosophical papers (Vol. I, pp. 26-39). New York: Oxford University Press.

Lewis, D. K. (1983b). Postscripts to "counterpart theory and quantified modal logic". In D. K. Lewis (Ed.), Philosophical papers (Vol. I, pp. 39-46). New York: Oxford University Press.

Lewis, D. K. (1983c). Counterparts of persons and their bodies. In D. K. Lewis (Ed.), Philosophical papers (Vol. I, pp. 47-54). New York: Oxford University Press.

Lewis, D. K. (1986a). On the plurality of worlds. Oxford: Blackwell.

Lewis, D. K. (1986b). Postscripts to "counterfactual dependence and time's arrow”. In D. K. Lewis (Ed.), Philosophical papers (Vol. II, pp. 52-66). New York: Oxford University Press.

Lewis, D. K. (1999a). New work for a theory of universals. In D. K. Lewis (Ed.), Papers in metaphysics and epistemology (pp. 8-55). Cambridge: Cambridge University Press.

Lewis D. K. (1999b). Against structural universals. In D. K. Lewis (Ed.), Papers in metaphysics and epistemology (pp. 78-107). Cambridge: Cambridge University Press.

Nolan, D. (2005). David Lewis. Bucks: Acumen.

${ }^{35}$ Lewis (1999a, pp. 16-17; 1986a, p. 13).

${ }^{36}$ See for instance Rodriguez-Pereyra (2002, pp. 91-92). 
Paul, L. A. (2004). The context of essence. In F. Jackson \& G. Priest (Eds.), Lewisian themes, the philosophy of David K. Lewis (pp. 181-195). Oxford: Clarendon Press.

Quine, W. V. O. (1961). Reference and modality. In W. V. O. Quine (Ed.), From a logical point of view (2nd ed., pp. 139-159). Cambridge: Harvard University Press.

Rodriguez-Pereyra, G. (2002). Resemblance nominalism: A solution to the problem of universals. Oxford: Oxford University Press.

Sider, T. (2011). Writing the book of the world. Oxford: Oxford University Press.

Taylor, B. (2006). Models, truth, and realism. Oxford: Oxford University Press. 